\title{
Insidious onset of intermittent claudication as the primary manifestation of infective endocarditis
}

This article was published in the following Dove Press journal:

International Journal of General Medicine

9 January 2017

Number of times this article has been viewed

\section{George Vasquez-Rios ${ }^{1,2}$ \\ Maria T Gamero ${ }^{2}$ \\ Jesus De la Cruz ${ }^{3}$ \\ Gabriel A Hernandez ${ }^{4}$ \\ Eduardo Hernandez ${ }^{5}$ \\ Roy Dueñas ${ }^{6}$}

'Alexander Von Humboldt Tropical Medicine Institute, Cayetano Heredia University, Lima, Peru; ${ }^{2}$ Alberto Hurtado School of Medicine, Cayetano Heredia University, Lima, Peru; Internal Medicine Department, Cayetano Heredia Hospital, Lima, Peru; ${ }^{4}$ Department of Cardiology, Miller School of Medicine, University of Miami, Miami, FL, ${ }^{5}$ Department of Adult Cardiology, St. Luke's Episcopal Hospital, Houston, TX, USA; ${ }^{6}$ Cardiology Department, Cayetano Heredia Hospital, Lima, Peru
Correspondence: George Vasquez-Rios Alexander Von Humboldt Tropical Medicine Institute, Cayetano Heredia University, Av. Honorio Delgado 430, San Martin de Porres I5I02, Peru Email george.vasquez@upch.pe

\begin{abstract}
Musculoskeletal manifestations of infective endocarditis are well-described in the literature. However, insidious onset of localized calf pain is an uncommon presentation of embolization and may represent a diagnostic challenge owing to the nonspecific nature of the symptoms. This study reviewed the literature and reports a case of infective endocarditis in a patient who presented with bilateral calf pain as the primary complaint and reason for seeking medical attention.
\end{abstract}

Keywords: musculoskeletal pain, embolization, ischemia, endocarditis

\section{Introduction}

Infective endocarditis (IE) presents with variable symptoms, including systemic or cardiovascular manifestations. ${ }^{1}$ Some of these symptoms are related to either embolic seeding of the organism or an immunological phenomenon associated with it. Although musculoskeletal symptoms are described in the literature, these are frequently overlooked by physicians..$^{2-3}$ In most reports, patients present with musculoskeletal manifestations resultant from acute limb ischemia, ${ }^{4-6}$ but more insidious presentations are rare. Failure to recognize this scenario may delay medical attention, contributing to a larger vegetation burden and increasing the mortality rates. Herein, a case is reported in which subacute bilateral calf pain was the primary manifestation of IE.

\section{Case presentation}

A 48-year-old man presented to the emergency department with 5 weeks of progressive intermittent claudication that was associated with low-grade fever and worsening dyspnea. His medical history was notable for tobacco use (five packs per day), heavy alcohol consumption, and cannabis use. He denied intravenous drug abuse and reported antibiotic use (amoxicillin) in the week prior to admission.

His vital signs were remarkable - a heart rate of $120 \mathrm{bpm}$, respiratory rate of $40 \mathrm{rpm}$, and oral temperature of $39^{\circ} \mathrm{C}$. On physical examination, breath sounds were decreased bilaterally and a 3/6 holosystolic murmur was perceived in the apex. Along with this, he had erythematous and swollen gingiva. No erythema or swelling was noted in the lower limbs, but capillary filling time was bilaterally delayed. Complete blood count revealed a white blood cell count of $15.4 \times 10^{3}$ cells $/ \mu \mathrm{L}(22 \%$ bands) and normocytic anemia with a hemoglobin level of $7.7 \mathrm{~g} / \mathrm{dL}$. Erythrocyte sedimentation rate and C-reactive protein were both increased at $94 \mathrm{~mm} / \mathrm{h}$ and $185 \mathrm{mg} / \mathrm{L}$, respectively. 
A

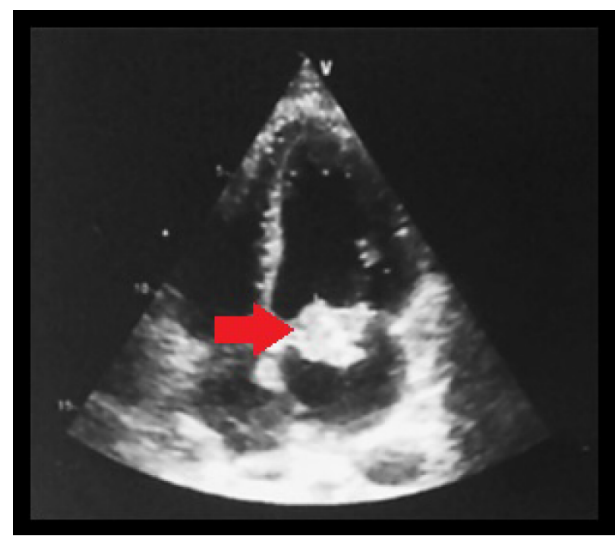

B

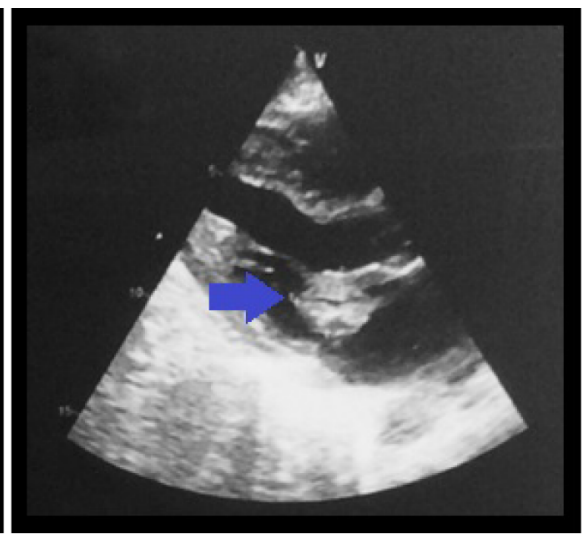

Figure I (A) Apical four-chamber view. (B) Long axis view.

Notes: (A) The red arrow shows the mass in the mitral valve during diastole. (B) The blue arrow shows a mass in the anterior leaflet of the mitral valve.

A chest X-ray showed bilateral pleural effusion, and the remaining biochemistry panel, urinalysis, and coagulation tests were within normal limits. Furthermore, serologic tests for HIV, viral hepatitis, and syphilis, in addition to immunologic studies (including antinuclear antibodies and anti-dsDNA) were negative. During evaluation, transthoracic echocardiography revealed a $2.1 \times 2 \mathrm{~cm}$ hyperechoic lesion on the anterior leaflet of the mitral valve (Figure 1A: a red arrow shows a mass in the apical four-chamber view. Figure 1B: a blue arrow shows the mass in the long axis view), which was associated with severe mitral regurgitation (regurgitant volume of $73 \mathrm{~mL}$ and vena contracta of $19 \mathrm{~mm}$ ) and an eccentrically directed posterior jet (despite jet angle, the effective regurgitation orifice was $>0.4 \mathrm{~cm}^{2}$ ). In addition, the ejection fraction was preserved. Similar findings were confirmed by transesophageal echocardiography. Furthermore, Doppler ultrasound of both inferior limbs showed bilateral arterial thrombosis in the right popliteal artery and in the left common femoral artery, with patency of collateral circulation. A subsequent computed tomography (CT) angiography of the lower extremities showed intraluminal filling defects in left common femoral and iliac artery (Figure 2A) and in the distal popliteal artery of both limbs (Figure 2B). The patient was started empirically on penicillin and gentamicin for possible infectious endocarditis. Blood culture results demonstrated no growth on the day of admission or during the follow-up (day 1, 3, and 5).

Despite the early use of broad-spectrum antibiotics for 4 days, he remained febrile and developed amaurosis fugax in the right eye, but CT scan of the head did not show evidence of an acute cerebrovascular event. His renal function declined, and the patient showed signs of hemodynamic instability with a mean arterial pressure of less than $60 \mathrm{mmHg}$. Thus, he was taken to the operating room on day 5 of hospitalization for

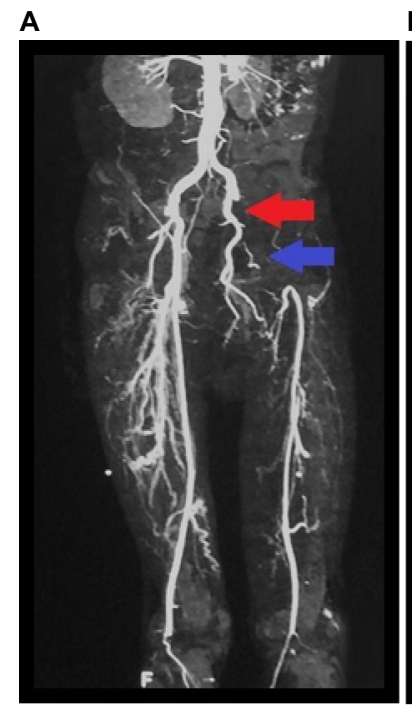

B

Figure 2 (A) CT angiography of lower extremities show intraluminal filling defects with occlusion of the left common iliac artery (red arrow) and left common femoral artery (blue arrow). (B) Intraluminal filling defect with occlusion in the distal popliteal artery of both limbs (red and blue arrows).

Abbreviation: $\mathrm{CT}$, computed tomography.

mitral valve debridement and valve replacement with a 27 mm size Carbomedics Orbis metallic valve (Sorin Group, Milan, Italy). After surgery, his fever subsided and clinical condition improved gradually. Histopathological and microbiologic studies (gram stain, bacterial and fungal cultures [Chocolate Agar, Mac Conkey agar, Heart-Brain Infusion agar, Czapek-Dox agar, and CHROMagar]) failed to find any organism. Considering his previous exposure to $\beta$-lactam medications and given the absence of etiologic risk factors other than his extensive gingival inflammation, he continued empiric treatment with ceftriaxone for a total of 4 weeks to cover Streptococci viridans, Staphylococcus aureus, and HACEK group organisms. Peripheral vascular procedures were not pursued due to the improvement of his bilateral calf pain and capillary filling time. Finally, the patient was placed 
on anticoagulation for his metallic valve and was enrolled in physical therapy. During follow-up (1, 3, and 6 months), he showed mild improvement in his bilateral claudication, and his overall status was stable.

\section{Discussion}

Musculoskeletal manifestations of IE, such as intermittent claudication, are described in the literature but are still poorly defined in medical practice. In a retrospective analysis including 192 cases of IE, 44\% of them presented one or more types of musculoskeletal manifestations (mainly arthralgia and low back pain) ${ }^{3}$ however, only $5.7 \%$ of the patients presented myalgia localized to the thigh or calf. In addition, $27 \%$ of the patients reported musculoskeletal complaints as one of the first symptoms of this disease. Furthermore, myalgia may be the sole manifestation of IE and can precede the diagnosis by as much as 6 weeks. ${ }^{2}$ No association has been noted between the severity of the rheumatic symptoms and laboratory test abnormalities. ${ }^{3}$

A number of embolic complications in IE have been reported in the literature. Previous reports have addressed acute embolic effects that may compromise limbs, ${ }^{5-7}$ spleen, or the central nervous system. ${ }^{8}$ Nonetheless, a subtle onset of musculoskeletal complaints, such as in this case, is rare and may lead to an adverse outcome if left unrecognized. The delayed clinical presentation of the patient may be partially explained by the compromise of small and medium-sized arteries as well as the progressive development of collateral circulation. In addition, it is possible that a certain degree of vascular damage was already present given his long-standing history of smoking.

Despite performing several blood cultures (6), no organism was isolated, possibly due to previous antibiotic use, which is the main cause of culture-negative endocarditis. ${ }^{9}$ Clinical guidelines recommend treatment with broadspectrum antibiotic coverage. ${ }^{9}$ However, on the basis of the local epidemiology, ${ }^{10}$ international data of IE, ${ }^{11}$ and taking into consideration the patient's risk factors (eg, extensive gingival inflammation and the lack of intravenous drug use), it was decided to prescribe ceftriaxone to treat Streptococci viridans. Appropriate antibiotic coverage is pivotal to prevent severe embolic events that may occur particularly during the first 2 weeks of the disease, ${ }^{12}$ especially if organisms such as Staphylococcus aureus, ${ }^{13}$ Streptococcus bovis, ${ }^{14}$ or fungi are involved. Additional factors such as vegetation characteristics (mobile vegetation length $>10 \mathrm{~mm}$, determined by transesophageal echocardiography $)^{9,15}$ may also be useful to estimate the real risk of further embolism, as in the patient (Box 1). Taking into account the aforementioned factors, a careful evaluation is needed when estimating the
Box I High risk for embolic events

Anterior mitral leaflet vegetation, particularly with size $10 \mathrm{~mm}$.
One embolic event during first 2 weeks of antimicrobial therapy.
Increase in vegetation size despite appropriate antimicrobial therapy.
Infection with particular microorganisms (Staphylococcus aureus,
Streptococcus bovis, Candida spp.)
Previous embolism (after diagnosis)
Multivalvular IE (bivalvular) involvement

Note: Data from Baddour et $\mathrm{al}^{9}$ and Habib et al. ${ }^{16}$

Abbreviation: IE, infective endocarditis.

real embolic burden as early surgical intervention may be warranted (recently acknowledged in the 2015 European guidelines for IE). ${ }^{16}$

Musculoskeletal manifestations of IE may appear early in the course of disease and may be a subtle clue to diagnosis. This case emphasizes the necessity of an early comprehensive examination and a broad differential diagnosis in patients with subacute vascular ischemic symptoms.

\section{Patient consent}

Written informed consent was obtained from the patient for publication of this case report and any accompanying images.

\section{Author contributions}

George Vasquez-Rios and Maria T Gamero wrote the manuscript and conducted the literature search. George VasquezRios, Maria T Gamero, Jesus de la Cruz, and Roy Dueñas were part of the medical team taking care of the patient. Gabriel A Hernandez and Eduardo Hernandez were consulted for expert opinion. George Vasquez-Rios, Maria T Gamero, Jesus de la Cruz, Gabriel A Hernandez, Eduardo Hernandez, and Roy Dueñas critically revised the manuscript for important intellectual consent. All authors contributed toward data analysis, drafting and critically revising the paper and agree to be accountable for all aspect of the work.

\section{Disclosure}

The authors report no conflicts of interest in this work.

\section{References}

1. Hoen B, Duval X. Infective endocarditis. N Engl J Med. 2013;368(15): 1425-1433.

2. Meyers OL, Commerford PJ. Musculoskeletal manifestations of bacterial endocarditis. Ann Rheum Dis. 1977;36(6):517-519.

3. Churchill MA Jr, Geraci JE, Hunder GG. Musculoskeletal manifestations of bacterial endocarditis. Ann Intern Med. 1977;87(6):754-759.

4. Pecoraro R, Tuttolomondo A, Parrinello G, Pinto A, Licata G. Staphylococcus lugdunensis endocarditis complicated by embolism in an 18-year-old woman with mitral valve prolapse. Case Rep Infect Dis. 2013;2013:4.

5. Lozano P, Flores D, Blanes I, et al. Acute lower limb ischemia complicating endocarditis due to Candida parapsilosis in a drug abuser. Ann Vasc Surg. 1994;8(6):591-594. 
6. Freischlag JA, Asbun HA, Sedwitz MM, Hye RJ, Sise M, Stabile BE. Septic peripheral embolization from bacterial and fungal endocarditis. Ann Vasc Surg. 1989;3(4):318-323.

7. Card L, Lofland D. Candidal endocarditis presenting with bilateral lower limb. Clin Lab Sci. 2012;25(3):130-134.

8. Thuny F, Di Salvo G, Belliard O, et al. Risk of embolism and death in infective endocarditis: prognostic value of echocardiography: a prospective multicenter study. Circulation. 2005;112(1):69-75.

9. Baddour LM, Wilson WR, Bayer AS, et al. Infective endocarditis: diagnosis, antimicrobial therapy, and management of complications: a statement for healthcare professionals from the Committee on Rheumatic Fever, Endocarditis, and Kawasaki Disease, Council on Cardiovascular Disease in the Young, and the Councils on Clinical Cardiology, Stroke, and Cardiovascular Surgery and Anesthesia, American Heart Association: endorsed by the Infectious Diseases Society of America. Circulation. 2005;111(23):e394-434.

10. Romane F, Cuadra J, Atencia F, Vargas F, Canelo C. Endocarditis infecciosa: análisis retrospectivo en el Hospital Nacional Arzobispo Loayza, 2002-2007. Rev Peru Epidemiol. 2009;13(2). Available from: http:// sisbib.unmsm.edu.pe/bvrevistas/epidemiologia/v13_n2/pdf/a04v13n2. pdf. Accessed April 3, 2015.
11. Slipczuk L, Codolosa JN, Davila CD, et al. Infective endocarditis epidemiology over five decades: a systematic review. PLoS One. 2013; 8(12):e82665.

12. Vuielle C, Nirdof M, Weyman AE, Picard MH. Natural history of vegetation during successful medical treatment of endocarditis. Am Heart J. 1994;128(6):1200-1209.

13. Erbel R, Liu F, Ge J, Rohmann S, Kupferwasser I. Identification of high-risk sub-groups in infective endocarditis and the role of echocardiography. Eur Heart J. 1995;16(5):588-602.

14. Pergola V, Di Salvo G, Habib G, et al. Comparison of clinical and echocardiographic characteristics of Streptococcus bovis endocarditis with that caused by other pathogens. Am J Cardiol. 2001;88(8): 871-875.

15. Tornos $P$, Iung $B$, Permanyer-Miralda $G$, et al. Infective endocarditis in Europe: lessons from the Euro heart survey. Heart. 2005;91(5):571-575.

16. Habib G, Lancellotti P, Antunes MJ, et al. 2015 ESC guidelines for the management of infective endocarditis: the task force for the management of infective endocarditis of the European Society of Cardiology (ESC) endorsed by: European Association for Cardio-Thoracic Surgery (EACTS), the European Association of Nuclear Medicine (EANM). Eur Heart J. 2015;36(44):3075-3128.
International Journal of General Medicine

\section{Publish your work in this journal}

The International Journal of General Medicine is an international, peer-reviewed open-access journal that focuses on general and internal medicine, pathogenesis, epidemiology, diagnosis, monitoring and treatment protocols. The journal is characterized by the rapid reporting of reviews, original research and clinical studies across all disease areas.

\section{Dovepress}

The manuscript management system is completely online and includes a very quick and fair peer-review system, which is all easy to use. Visit http://www.dovepress.com/testimonials.php to read real quotes from published authors. 